\title{
Erratum to: Identifying Influential Spreaders by Graph Sampling
}

Nikos Salamanos, Elli Voudigari and Emmanuel J. Yannakoudakis

Erratum to:

Chapter "Identifying Influential Spreaders by Graph Sampling" in: H. Cherifi et al. (eds.), Complex Networks \& Their Applications V, Studies in Computational Intelligence 693, DOI 10.1007/978-3-319-50901-3_9

The original version of the book was inadvertently published without the following corrections:

1. The Figs. 2-4 wrongly placed after the paper references should be placed before Sect. 5 Conclusion in pages 118-120.

2. The "Algorithm-3" in pages $113 \& 114$ should be changed to read as "Algorithm-1".

3. In page 113, after Step-10, the numbering of the algorithmic steps should be corrected to match the original version and inside Algorithm, Steps-2 and 13 have wrong equation alignment to be corrected.

4. In page 116, Table 1, first row, second column: spacing problem in the words "egoFacebook" and "wiki-Vote" should be cleared.

5. Almost all sub-figure links leading to other papers of the proceedings should be changed to lead to correct figures of the paper.

Specifically, the links, in Sect. 4.3:

Fig. 1a, page-116, line-14

Fig. $1 \mathrm{~b}$ and 1c, page-116, line-14

Fig. 2a, page-116, line-19

Fig. 2b, page-116, line-21

The updated original online version for this chapter can be found at http://dx.doi.org/10.1007/978-3-319-50901-3_9 
Fig. 2c, page-116, line-22

Fig. $3 a$ and $3 b$, page- 117 , line- 12

Fig. 3d, page-118, line-3 